\title{
ON THE INVERSE TO THE HARMONIC OSCILLATOR
}

\author{
MARCO CAPPIELLO, LUIGI RODINO, AND JOACHIM TOFT
}

\begin{abstract}
Let $b_{d}$ be the Weyl symbol of the inverse to the harmonic oscillator on $\mathbf{R}^{d}$. We prove that $b_{d}$ and its derivatives satisfy convenient bounds of Gevrey and Gelfand-Shilov type, and obtain explicit expressions for $b_{d}$. In the even-dimensional case we characterize $b_{d}$ in terms of elementary functions.

In the analysis we use properties of radial symmetry and a combination of different techniques involving classical a priori estimates, commutator identities, power series and asymptotic expansions.
\end{abstract}

\section{INTRODUCTION}

A fundamental operator in quantum physics and classical analysis is the harmonic oscillator

$$
H=H_{d}=-\Delta+|x|^{2}, \quad x \in \mathbf{R}^{d} .
$$

In physics the operator $H$ appears in the stationary Schrödinger equation for a particle under the action of a quadratic potential. In classical analysis, $H$ is also known as the Hermite operator, and possesses several convenient properties. For example, the operator $H$ is strictly positive in $L^{2}\left(\mathbf{R}^{d}\right)$ with discrete spectrum, and the eigenfunctions are the Hermite functions, see for example [7, 15, 19].

By means of the Hermite functions one can express also the kernel of the inverse $H^{-1}$. On the other hand, coherently with the point of view of the quantum physics, $H^{-1}$ can be written as Weyl pseudo-differential operator

$$
H^{-1} f(x)=(2 \pi)^{-d} \iint e^{i\langle x-y, \xi\rangle} b((x+y) / 2, \xi) f(y) d y d \xi
$$

for a suitable symbol $b(x, \xi)=b_{d}(x, \xi)$ in $\mathbf{R}^{2 d}$ (see for example the general calculus in $[9,10,12,16$, for classes of symbols and operators in $\mathbf{R}^{d}$ ). The calculus provides, as a particular case, the construction in these classes of the symbol of the parametrix of $H$. Also the symbol of the inverse $b_{d}(x, \xi)$ in (0.2) belongs to the same classes, in view of the property of spectral invariance (cf. [1]). Despite the power of

2010 Mathematics Subject Classification. primary 35Q40; 35S05; 46F05; secondary 33C10; 30Gxx.

Key words and phrases. harmonic oscillator, inverse, Gelfand-Shilov estimates, ultradistributions. 
the pseudo-differential theory, the study of the peculiar properties of $b_{d}(x, \xi)$ is missing in literature.

The aim of the paper is to analyze the function $b_{d}(x, \xi)$ in $\mathbf{R}^{2 d}$ and to derive suitable regularity estimates and explicit expressions. In the even-dimensional case we express $b_{d}$ in terms of elementary functions. Just to have a sample of our study, we here mention the striking and seemingly unnoticed formula in dimension $d=2$ :

$$
b_{2}(x, \xi)=\frac{1-e^{-|x|^{2}-|\xi|^{2}}}{|x|^{2}+|\xi|^{2}} .
$$

Before giving a more detailed presentation of our results, we recall some known facts for $H$. First, we have convenient bijectivity properties of $H$ in different function, distribution and ultradistribution spaces. (See e. g. 99 12, 14, 16], and Proposition 2.2 and Theorem 3.10 in [17.) Furthermore, the operator possesses useful regularity properties. For example, if $f \in \mathscr{S}^{\prime}\left(\mathbf{R}^{d}\right)$, and

$$
H f \in \mathscr{S}\left(\mathbf{R}^{d}\right) \quad \text { or } \quad H^{N} f \in L^{2}\left(\mathbf{R}^{d}\right)
$$

for every $N$, then $f \in \mathscr{S}\left(\mathbf{R}^{d}\right)$. One way to obtain the latter property is to use Theorem 3.10 in [17]. The other standard way is to use Theorem 25.4 (with $m=m_{0}=2$ ) in [16], which implies that

$$
\left|\partial^{\alpha} b_{d}(x, \xi)\right| \leq C_{\alpha}\langle(x, \xi)\rangle^{-2-|\alpha|},
$$

and using appropriate mapping properties of pseudo-differential operators with symbols satisfying (0.4). In 3] more refined estimates are established for the solutions $f \in \mathscr{S}^{\prime}\left(\mathbf{R}^{d}\right)$ of the equation $H f=g$ and for more general differential operators when $g$ belongs to the GelfandShilov space $\mathcal{S}_{s}\left(\mathbf{R}^{d}\right), s \geq 1 / 2$ (cf. Section 1 for the definitions).

The first aim of this paper is to establish certain refinements as well as other estimates related to (0.4). Imitating the local analytic calculus of [2] and trying to adapt the global Gelfand-Shilov calculus of [6] one could tentatively assume

$$
\left|\partial^{\alpha} b_{d}(x, \xi)\right| \leq C^{|\alpha|+1}(\alpha !)^{s_{0}}\langle(x, \xi)\rangle^{-2-|\alpha|},
$$

for some $s_{0} \geq 1 / 2$ and positive constant $C$ independent of $\alpha$. A global calculus for symbols satisfying factorial estimates of the form (0.5) does not exist in the literature, especially for $1 / 2 \leq s_{0}<1$, and sharp estimates for $b_{d}$ are considered as an open and difficult problem. Nevertheless in the present paper we prove that the estimate

$$
\left|\partial^{\alpha} b_{d}(x, \xi)\right| \leq C^{|\alpha|+1}(\alpha !)^{(s+1) / 2}\langle(x, \xi)\rangle^{-2-s|\alpha|},
$$

holds for some positive constant $C$ which is independent of $\alpha \in \mathbf{N}^{2 d}$ and $s \in[0,1]$. In particular, for $s=0$ we have

$$
\left|\partial^{\alpha} b_{d}(x, \xi)\right| \leq C^{|\alpha|+1}(\alpha !)^{1 / 2}\langle(x, \xi)\rangle^{-2},
$$


whereas, for $s=1$, (0.4) is refined into

$$
\left|\partial^{\alpha} b_{d}(x, \xi)\right| \leq C^{|\alpha|+1} \alpha !\langle(x, \xi)\rangle^{-2-|\alpha|},
$$

for some constant $C$ which is independent of $\alpha \in \mathbf{N}^{2 d}$. Furthermore, we use (0.6) to establish similar estimates for $b_{d, t}$, the $t$-symbol of $H^{-1}$ (cf. Theorem 2.1, Proposition 2.2 and Remarks 2.4 and 2.5).

Starting from the estimate (0.6) for $b_{d}$, it might be interesting to study general symbols satisfying estimates of the same type and to establish regularity results for the related operators in the setting of Gelfand-Shilov spaces as it has been done in [3 5]. We will treat these applications in future papers and focus here only on the model $\mathrm{H}^{-1}$.

To prove (0.6), in Section 2 we use classical a priori estimates for globally elliptic operators, and suitable commutator estimates. Moreover we apply the symbolic calculus to prove that $b_{d}$ satisfies

$$
\left(H_{0} b_{d}\right)(X) \equiv|X|^{2} b_{d}(X)-\frac{1}{4} \Delta_{X} b_{d}(X)=1, \quad X=(x, \xi) \in \mathbf{R}^{2 d} .
$$

We note that the operator on the left-hand side is a (dilated) harmonic oscillator on the phase space variables $X=(x, \xi) \in \mathbf{R}^{2 d}$. In Sections 1 and 2 we show that any solution of (0.9) is of the form

$$
b_{d}(X)=c_{d}\left(|X|^{2}\right) \text {, }
$$

for some entire function $c_{d}$ on $\mathbf{C}$. In particular, $b_{d}$ is radial symmetric.

This introduces the next main issue, which concerns explicit formulas for $b_{d}$, and is presented in Section 3. In fact, by the radial symmetry we may reduce (0.9) into an ordinary differential equation on $c_{d}$. A power series expansion and (0.7) then give

$$
\begin{aligned}
& c_{d}(t) \\
& =\frac{d ! !}{d}\left(\alpha \sum_{p=0}^{\infty} \frac{t^{2 p}}{(2 p) ! !(2 p+d-1) ! !}-\sum_{p=0}^{\infty} \frac{t^{2 p+1}}{(2 p+1) ! !(2 p+d) ! !}\right),
\end{aligned}
$$

where $\alpha$ is equal to 1 when $d$ is even, and equal to $\pi / 2$ when $d$ is odd.

The expansion (0.11) gives finite analytic expressions for $c_{d}$, thereby for $b_{d}$, when $d=2 n$ is even. Namely, we first obtain an explicit asymptotic expansion of the symbol $b_{d}$ in terms of homogeneous functions in $|(x, \xi)|$. Then, by a slight modification of this expansion inspired by (0.3), we establish the general formula

$$
b_{2 n}(x, \xi)=\sum_{j=0}^{n-1}\left(\begin{array}{c}
n-1 \\
j
\end{array}\right)(-1)^{j}(2 j) ! \frac{1-e^{-|x|^{2}-|\xi|^{2}} p_{2 j}\left(|x|^{2}+|\xi|^{2}\right)}{\left(|x|^{2}+|\xi|^{2}\right)^{2 j+1}}
$$

for the symbol $b_{2 n}(x, \xi)$, where $p_{2 j}(t)$ denotes the Taylor polynomial of $e^{t}$ of order $2 j$ centered at $t=0$ (cf. formula (3.27) in Section 31).

For the odd dimensional case, the formula (0.11) does not give any simple expressions of finite numbers of elementary functions. In fact, if 
$d=2 n+1$, then the first series in (0.11) is equal to $u_{n}\left(t^{2} / 4\right)$, for some Bessel function $u_{n}$ (cf. Theorem 3.2), and for the second series it seems to be even more complicated to find well-known special functions, since each coefficient contains two factors of odd semi-factorials. For example, in contrast to the the first series, the second one can not be completely described by Bessel functions. On the other hand, by the link between $c_{d}$ and $b_{d}$, a combination of (0.6) and (0.11) leads to

$$
\sum_{p=0}^{\infty} \frac{t^{2 p+1}}{(2 p+1) ! !(2 p+d) ! !}=\frac{\pi}{2} u_{n}\left(t^{2} / 4\right)+\mathcal{O}(1 / t), \quad t \rightarrow+\infty
$$

which seems to be unknown until now and should be interesting in the theory of special functions. (See Theorem 3.2 and Remark 3.3 for more detailed explanations, which also include more detailed estimates for the involved functions and their derivatives).

\section{Preliminaries}

In this section we recall some basic results on pseudo-differential calculus. We shall often formulate these results in the framework of the Gelfand-Shilov space $\mathcal{S}_{1 / 2}\left(\mathbf{R}^{d}\right)$ and its dual $\mathcal{S}_{1 / 2}^{\prime}\left(\mathbf{R}^{d}\right)$ (see e. g. [8]). The reader who is not interested in this general situation may replace $\mathcal{S}_{1 / 2}\left(\mathbf{R}^{d}\right)$ and $\mathcal{S}_{1 / 2}^{\prime}\left(\mathbf{R}^{d}\right)$ by $\mathscr{S}\left(\mathbf{R}^{d}\right)$ and $\mathscr{S}^{\prime}\left(\mathbf{R}^{d}\right)$ respectively. Here $\mathscr{S}\left(\mathbf{R}^{d}\right)$ is the set of Schwartz functions on $\mathbf{R}^{d}$, and $\mathscr{S}^{\prime}\left(\mathbf{R}^{d}\right)$ is the set of tempered distributions on $\mathbf{R}^{d}$, see e.g. [10].

We start by recalling the definition of Gelfand-Shilov spaces. Let $s \geq 1 / 2$ be fixed. For any $f \in C^{\infty}\left(\mathbf{R}^{d}\right)$ and $h>0$ we let

$$
\|f\|_{\mathcal{S}_{s, h}} \equiv \sup \frac{\left|x^{\beta} \partial^{\alpha} f(x)\right|}{h^{|\alpha|+|\beta|}(\alpha ! \beta !)^{s}}
$$

Here the supremum should be taken over all $x \in \mathbf{R}^{d}$ and multi-indices $\alpha, \beta \in \mathbf{N}^{d}$. Then the Gelfand-Shilov space $\mathcal{S}_{s}\left(\mathbf{R}^{d}\right)$ consists of all $f \in$ $C^{\infty}\left(\mathbf{R}^{d}\right)$ such that $\|f\|_{\mathcal{S}_{s, h}}$ is finite for some $h>0$. Evidently, $\mathcal{S}_{s}\left(\mathbf{R}^{d}\right) \subset$ $\mathscr{S}\left(\mathbf{R}^{d}\right)$ for every $s \geq 1 / 2$.

The set $\mathcal{S}_{s}\left(\mathbf{R}^{d}\right)$ contains all finite linear combinations of Hermite functions. Since such linear combinations are dense in $\mathscr{S}\left(\mathbf{R}^{d}\right)$, it follows that the dual $\mathcal{S}_{s}^{\prime}\left(\mathbf{R}^{d}\right)$ of $\mathcal{S}_{s}\left(\mathbf{R}^{d}\right)$ is a space which contains $\mathscr{S}^{\prime}\left(\mathbf{R}^{d}\right)$.

We refer to [8,12] for more facts about Gelfand-Shilov spaces and their duals.

Next we recall certain properties of pseudo-differential operators. Let $t \in \mathbf{R}$. For any $a \in \mathcal{S}_{1 / 2}\left(\mathbf{R}^{2 d}\right)$, the pseudo-differential operator $\mathrm{Op}_{t}(a)$ is the linear and continuous operator on $\mathcal{S}_{1 / 2}\left(\mathbf{R}^{d}\right)$, defined by

$$
\mathrm{Op}_{t}(a) f(x)=(2 \pi)^{-d / 2} \iint_{\mathbf{R}^{2 d}} a((1-t) x+t y, \xi) f(y) e^{i\langle x-y, \xi\rangle} d y d \xi
$$


The definition extends uniquely to any $a \in \mathcal{S}_{1 / 2}^{\prime}\left(\mathbf{R}^{2 d}\right)$, and then $\mathrm{Op}_{t}(a)$ is continuous from $\mathcal{S}_{1 / 2}\left(\mathbf{R}^{d}\right)$ to $\mathcal{S}_{1 / 2}^{\prime}\left(\mathbf{R}^{d}\right)$. (Cf. [18].) In the case $t=0$, then $\mathrm{Op}_{0}(a)$ agrees with the Kohn-Nirenberg representation $a(x, D)$, and if $t=1 / 2$, then $\mathrm{Op}_{1 / 2}(a)$ is equal to the Weyl quantization $\mathrm{Op}^{w}(a)$.

Now we recall the definition of the Shubin class of pseudo-differential operators. Let $m \in \mathbf{R}$. Then the Shubin class $\Gamma^{m}\left(\mathbf{R}^{2 d}\right)$ is the set of all functions $a(x, \xi) \in C^{\infty}\left(\mathbf{R}^{2 d}\right)$ satisfying the estimate

$$
\left|\partial^{\alpha} a(x, \xi)\right| \leq C_{\alpha}\langle(x, \xi)\rangle^{m-|\alpha|}, \quad(x, \xi) \in \mathbf{R}^{2 d} .
$$

In particular, for the symbols of the harmonic oscillator and its inverse, we have $h \in \Gamma^{2}\left(\mathbf{R}^{2 d}\right)$ and $b_{d} \in \Gamma^{-2}\left(\mathbf{R}^{2 d}\right)$. By (23.17) in [16], the operators $\mathrm{Op}_{t}(a)$ with $a \in \Gamma^{m}\left(\mathbf{R}^{2 d}\right)$ are continuous on $\mathscr{S}\left(\mathbf{R}^{d}\right)$, and on $\mathscr{S}^{\prime}\left(\mathbf{R}^{d}\right)$.

A symbol $a \in \Gamma^{m}\left(\mathbf{R}^{2 d}\right)$ is said to be globally elliptic if

$$
|a(x, \xi)| \geq c|(x, \xi)|^{m}, \quad \text { when } \quad|(x, \xi)| \geq R,
$$

for some positive constants $c$ and $R$.

In the following we shall prove a result on the radial symmetry of solutions of the problem

$$
\mathrm{Op}_{t}(a) f=g
$$

where $\mathrm{Op}_{t}(a)$ is the pseudo-differential operator given by (1.1). Here we recall that an element $f \in \mathcal{S}_{1 / 2}^{\prime}\left(\mathbf{R}^{d}\right)$ is called radial symmetric, if the pullback $U^{*} f$ is equal to $f$, for every unitary transformation $U$ on $\mathbf{R}^{d}$. In the case when $f$ in addition is a measurable function, then $f$ is radial symmetric, if and only if $f(x)=f_{0}(|x|)$ a. e., for some measurable function $f_{0}$ on $\mathbf{R}$.

Proposition 1.1. Let $a \in \mathcal{S}_{1 / 2}^{\prime}\left(\mathbf{R}^{2 d}\right)$ and $f, g \in \mathcal{S}_{1 / 2}^{\prime}\left(\mathbf{R}^{d}\right)$ be such that $\mathrm{Op}_{t}(a) f$ is well defined and is equal to $g$, and that $a(x, \xi)$ is a radial symmetric symbol in the $x$-variable and in the $\xi$-variable. Then the following is true:

(1) If $f$ is radial symmetric, then $g$ is radial symmetric;

(2) If $\mathrm{Op}_{t}(a)$ is injective and $g$ is radial symmetric, then $f$ is radial symmetric. 
Proof. Assume that $f$ is radial symmetric and let $U$ be a unitary matrix on $\mathbf{R}^{d}$. Then formal computations give

$$
\begin{aligned}
g(U x)= & (2 \pi)^{-d} \iint a((1-t) U x+t y, \xi) f(y) e^{i\langle U x-y, \xi\rangle} d y d \xi \\
= & (2 \pi)^{-d} \iint a((1-t) U x+t y, U \xi) f(y) e^{i\left\langle x-U^{-1} y, \xi\right\rangle} d y d \xi \\
= & (2 \pi)^{-d} \iint a(U((1-t) x+t y), \xi) f(y) e^{i\langle x-y, \xi\rangle} d y d \xi \\
= & (2 \pi)^{-d} \iint a((1-t) x+t y, \xi) f(y) e^{i\langle x-y, \xi\rangle} d y d \xi=g(x),
\end{aligned}
$$

which proves that $g$ is radial symmetric. Hence (1) holds.

Assume instead that $g$ is radial symmetric and that $\mathrm{Op}_{t}(a)$ is injective. Again let $U$ be an arbitrary unitary matrix. By (1.3) we have

$$
\begin{array}{r}
g(x)=g(U x)=(2 \pi)^{-d} \iint a((1-t) U x+t y, \xi) f(y) e^{i\langle U x-y, \xi\rangle} d y d \xi \\
=(2 \pi)^{-d} \iint a(U((1-t) x+t y), U \xi) f(U y) e^{i\langle U(x-y), U \xi\rangle} d y d \xi \\
=(2 \pi)^{-d} \iint a((1-t) x+t y, \xi) f(U y) e^{i\langle x-y, \xi\rangle} d y d \xi .
\end{array}
$$

Hence both $f$ and $U^{*} f$ solves (1.3). Since $\operatorname{Op}(a)$ is injective, it follows that $f=U^{*} f$. Consequently, $f$ is radial symmetric, and (2) follows. The proof is complete.

Proposition 1.1 applies in particular to the harmonic oscillator giving the following result.

Corollary 1.2. Let $f, g \in \mathcal{S}_{1 / 2}^{\prime}\left(\mathbf{R}^{d}\right)$ be such that

$$
\left(-\Delta+C|x|^{2}\right) f=g
$$

for some constant $C>0$. Then $f$ is radial symmetric if and only if $g$ is radial symmetric.

Remark 1.3. In the literature it is common to add a constant (the spectral parameter) to the harmonic oscillator and to consider the more general equation

$$
\left(-\Delta+C_{1}|x|^{2}+C_{2}\right) f=g .
$$

For example, the Helmholz equation is of this form.

In particular, Corollary 1.2 can be extended into the following. Let $f, g \in \mathcal{S}_{1 / 2}^{\prime}\left(\mathbf{R}^{d}\right)$ be such that $(1.4)^{\prime}$ holds for some constants

$$
C_{1}>0 \quad \text { and } \quad C_{2} \in \mathbf{C} \backslash\left\{C_{1}^{1 / 2}(-d-2 n) ; n \in \mathbf{N}\right\} .
$$

Then $f$ is radial symmetric if and only if $g$ is radial symmetric. 


\section{ESTimates FOR THE INVERSE OF THE HARMONIC OSCILlATOR}

In this section we derive estimates for the Weyl symbol of the inverse to the harmonic oscillator. In the last part of the section we shall use these results to obtain related estimates for the $t$-symbol of that inverse. More precisely, we prove the following result, which in the case $s=$ 1 gives more detailed information about $b_{d}$ compared to the Shubin estimate (0.4).

Theorem 2.1. Let $b_{d}$ be the Weyl symbol of the inverse to the harmonic oscillator on $\mathbf{R}^{d}$. Then there is a constant $C>0$ such that (0.6) holds for every $\alpha \in \mathbf{N}^{2 d}$ and $s \in[0,1]$.

By an argument with geometric mean-values, it suffices to prove the result in the limit cases $s=0$ and $s=1$, which correspond to the estimates (0.7) and (0.8), respectively, Since these cases are interesting by their own we write them as two independent statements.

Proposition 2.2. Let $b_{d}$ be the Weyl symbol of the inverse to the harmonic oscillator on $\mathbf{R}^{d}$. Then there is a constant $C>0$ such that (0.7) holds for every $\alpha \in \mathbf{N}^{2 d}$.

Proposition 2.3. Let $b_{d}$ be the Weyl symbol of the inverse to the harmonic oscillator on $\mathbf{R}^{d}$. Then there is a constant $C>0$ such that (0.8) holds for every $\alpha \in \mathbf{N}^{2 d}$.

In order to prove Proposition 2.2 we need some preparation. The invertibility properties and the symbolic calculus give

$$
\left(|x|^{2}+|\xi|^{2}\right) \# b_{d}(x, \xi)=1,
$$

where \# is the Weyl product (cf. Section 18.5 in [10]). We claim that

$$
H_{0} b_{d}(x, \xi) \equiv\left(|x|^{2}+|\xi|^{2}\right) b_{d}(x, \xi)-\frac{1}{4} \Delta_{x, \xi} b_{d}(x, \xi)=1
$$

and

$$
\sum_{j=1}^{d}\left(x_{j} \partial_{\xi_{j}} b_{d}(x, \xi)-\xi_{j} \partial_{x_{j}} b_{d}(x, \xi)\right)=0 .
$$

In fact, by (2.1) and asymptotic expansion we get

$$
H_{0} b_{d}(x, \xi)+i \sum_{j=1}^{d}\left(x_{j} \partial_{\xi_{j}} b_{d}(x, \xi)-\xi_{j} \partial_{x_{j}} b_{d}(x, \xi)\right)=1 .
$$

Since $H$ is self-adjoint, it follows that $\mathrm{Op}^{w}\left(b_{d}\right)$ is also self-adjoint. By using the fact that a Weyl operator is self-adjoint, if and only if its Weyl symbol is real-valued, it follows that $b_{d}$ is real-valued. Hence (2.4) gives (2.2) and (2.3). 
Proof of Proposition 2.2. For $\alpha=0$ the assertion is true since $b_{d}$ is in $\Gamma^{-2}\left(\mathbf{R}^{2 d}\right)$. Assume instead that $\alpha \neq 0$. By letting $X=(x, \xi)$, it follows that (2.2) is the same as (0.9). Since $H_{0}$ is globally elliptic, we have

$$
\sum_{|\gamma+\delta| \leq 2}\left\|X^{\gamma} \partial_{X}^{\delta} u\right\|_{L^{p}\left(\mathbf{R}^{2 d}\right)} \leq C_{p}\left\|H_{0} u\right\|_{L^{p}\left(\mathbf{R}^{2 d}\right)}
$$

for every $p \in(1, \infty), u \in \mathscr{S}\left(\mathbf{R}^{2 d}\right)$ and for some constant $C_{p}$ depending on $p$ and $d$ only (cf. [12]). From now on, let $p>2 d$. With this choice, $b_{d}(X) \in L^{p}\left(\mathbf{R}^{2 d}\right)$ together with all its derivatives and the same holds for $\langle X\rangle b_{d}(X)$, since $b_{d} \in \Gamma^{-2}\left(\mathbf{R}^{2 d}\right)$. Now let $u=\partial_{X}^{\alpha} b_{d}$ in (2.5), where $\alpha \in \mathbf{N}^{2 d} \backslash\{0\}$. In order to obtain appropriate estimates we consider the commutator

$$
\left[H_{0}, \partial_{X}^{\alpha}\right] b_{d}=H_{0}\left(\partial_{X}^{\alpha} b_{d}\right)-\partial_{X}^{\alpha}\left(H_{0} b_{d}\right)=H_{0}\left(\partial_{X}^{\alpha} b_{d}\right)
$$

since $H_{0} b_{d}=1$. By combining (2.5) and (2.6) we get

$$
\sum_{|\gamma+\delta| \leq 2}\left\|X^{\gamma} \partial_{X}^{\delta+\alpha} b_{d}\right\|_{L^{p}\left(\mathbf{R}^{2 d}\right)} \leq C\left\|\left[H_{0}, \partial_{X}^{\alpha}\right] b_{d}\right\|_{L^{p}\left(\mathbf{R}^{2 d}\right)}
$$

Since $\left[\Delta_{X}, \partial_{X}^{\alpha}\right]=0$, we have

$$
\begin{aligned}
{\left[H_{0}, \partial_{X}^{\alpha}\right] b_{d} } & =\left[|X|^{2}, \partial_{X}^{\alpha}\right] b_{d} \\
& =-2 \sum_{\substack{1 \leq j \leq 2 d \\
\alpha_{j} \neq 0}} \alpha_{j} X_{j} \partial_{X}^{\alpha-e_{j}} b_{d}-\sum_{\substack{1 \leq j \leq 2 d \\
\alpha_{j} \geq 2}} \alpha_{j}\left(\alpha_{j}-1\right) \partial_{X}^{\alpha-2 e_{j}} b_{d}
\end{aligned}
$$

where $e_{j}, j=1, \ldots, 2 d$, is the standard basis in $\mathbf{R}^{2 d}$.

Now we set, for $\alpha \neq 0$ :

$$
J_{\alpha}=\sum_{\substack{|\gamma+\delta| \leq 2 \\(\gamma, \delta) \neq(0,0)}}\left\|X^{\gamma} \partial_{X}^{\delta+\alpha} b_{d}\right\|_{L^{p}\left(\mathbf{R}^{2 d}\right)}
$$


From (2.7) and (2.8) it follows that

$$
\begin{gathered}
J_{\alpha} \leq 2 C \sum_{\substack{1 \leq j \leq 2 d \\
\alpha_{j} \neq 0}} \alpha_{j}\left\|X_{j} \partial_{X}^{\alpha-e_{j}} b_{d}\right\|_{L^{p}\left(\mathbf{R}^{2 d}\right)}+C \sum_{\substack{1 \leq j \leq 2 d \\
\alpha_{j} \geq 2}} \alpha_{j}\left(\alpha_{j}-1\right)\left\|\partial_{X}^{\alpha-2 e_{j}} b_{d}\right\|_{L^{p}\left(\mathbf{R}^{2 d}\right)} \\
=2 C \sum_{\substack{1 \leq j \leq 2 d \\
\alpha_{j}=1}}\left\|X_{j} \partial^{\alpha-e_{j}} b_{d}\right\|_{L^{p}\left(\mathbf{R}^{2 d}\right)}+2 C \sum_{\substack{1 \leq j \leq 2 d \\
\alpha_{j} \geq 2}} \alpha_{j}\left\|X_{j} \partial_{j} \partial^{\alpha-2 e_{j}} b_{d}\right\|_{L^{p}\left(\mathbf{R}^{2 d}\right)} \\
+C \sum_{\substack{1 \leq j \leq 2 d \\
2 \leq \alpha_{j} \leq 3}} \alpha_{j}\left(\alpha_{j}-1\right)\left\|\partial^{\alpha-2 e_{j}} b_{d}\right\|_{L^{p}\left(\mathbf{R}^{2 d}\right)}+C \sum_{\substack{1 \leq j \leq 2 d \\
\alpha_{j} \leq 4}} \alpha_{j}\left(\alpha_{j}-1\right)\left\|\partial_{j}^{2} \partial^{\alpha-4 e_{j}} b_{d}\right\|_{L^{p}\left(\mathbf{R}^{2 d}\right)} \\
\leq 2 C \sum_{\substack{1 \leq j \leq 2 d \\
\alpha_{j}=1}} J_{\alpha-e_{j}}+2 C \sum_{\substack{1 \leq j \leq 2 d \\
\alpha_{j} \geq 2}} \alpha_{j} J_{\alpha-2 e_{j}} \\
+6 C \sum_{\substack{1 \leq j \leq 2 d \\
2 \leq \alpha_{j} \leq 3}} J_{\alpha-2 e_{j}}+C \sum_{\substack{1 \leq j \leq 2 d \\
\alpha_{j} \geq 4}} \alpha_{j}\left(\alpha_{j}-1\right) J_{\alpha-4 e_{j}}, \quad(2.9)
\end{gathered}
$$

Using (2.9) we want now to prove by induction on $|\alpha| \geq 1$ that

$$
J_{\alpha} \leq C_{1}^{|\alpha|}(\alpha !)^{1 / 2}
$$

for some positive constant $C_{1}$ depending only on $d$ and on the constant $C=C_{p}$ in (2.5). For $|\alpha| \leq 4, \alpha \neq 0$, the assertion is obvious. Now assume that it is true for $|\alpha| \leq N-1$ and let us prove it for $|\alpha|=N$. We observe that

$$
\alpha_{j}\left(\alpha-2 e_{j}\right) !^{1 / 2} \leq \sqrt{2}\left(\alpha_{j}\left(\alpha_{j}-1\right)\left(\alpha-2 e_{j}\right) !\right)^{1 / 2}=\sqrt{2}(\alpha !)^{1 / 2}
$$

and

$$
\begin{array}{r}
\alpha_{j}\left(\alpha_{j}-1\right)\left(\alpha-4 e_{j}\right) !^{1 / 2} \leq 2\left(\alpha_{j}\left(\alpha_{j}-1\right)\left(\alpha_{j}-2\right)\left(\alpha_{j}-3\right)\left(\alpha-4 e_{j}\right) !\right)^{1 / 2} \\
=2(\alpha !)^{1 / 2}
\end{array}
$$

Then, from (2.9) and from the inductive assumption we obtain

$$
\begin{aligned}
J_{\alpha} \leq & 4 C d C_{1}^{|\alpha|-1}(\alpha !)^{1 / 2}+4 \sqrt{2} C d C_{1}^{|\alpha|-2}(\alpha !)^{1 / 2} \\
& +12 C d C_{1}^{|\alpha|-2}(\alpha !)^{1 / 2}+4 C d C_{1}^{|\alpha|-4}(\alpha !)^{1 / 2} \leq C_{1}^{|\alpha|}(\alpha !)^{1 / 2}
\end{aligned}
$$

choosing $C_{1}$ sufficiently large. In particular, from (2.10) we obtain that

$$
\sum_{|\gamma| \leq 2}\left\|X^{\gamma} \partial_{X}^{\alpha} b_{d}\right\|_{L^{p}\left(\mathbf{R}^{2 d}\right)} \leq C_{1}^{|\alpha|+1}(\alpha !)^{1 / 2}
$$

holds for every $\alpha \in \mathbf{N}^{2 d}, \alpha \neq 0$. Finally, the estimate (0.7) follows from standard Sobolev embedding estimates. 
Proof of Proposition 2.3. In view of Proposition 2.2, it is sufficient to prove the estimate (0.8) for $|X| \geq 1$. First we prove that if $p>2 d$, then $b_{d}$ satisfies the following estimate

$$
\left\|X^{\beta+\tau} \partial_{X}^{\alpha} b_{d}\right\|_{L^{p}\left(\mathbf{R}^{2 d}\right)} \leq C_{o}^{|\alpha|+1}|\alpha|^{|\alpha|}, \quad X \in \mathbf{R}^{2 d}
$$

for every $\alpha, \beta, \tau \in \mathbf{N}^{2 d}$ with $\alpha \neq 0,|\beta|<|\alpha|$ and $|\tau| \leq 2$. Namely, setting $M=|\alpha+\beta+\tau|$, we shall obtain (2.11) by proving the following estimate

$$
\left\|X^{\beta+\tau} \partial_{X}^{\alpha} b_{d}\right\|_{L^{p}\left(\mathbf{R}^{2 d}\right)} \leq C^{M+1} M^{M}
$$

for some positive constant $C$ independent of $M$. We shall argue by induction on $M$. For $M \leq 4$, the estimate (2.12) holds true since by the Shubin estimate (0.5) we have

$$
\left|X^{\beta+\tau} \partial_{X}^{\alpha} b_{d}(X)\right| \leq C_{1}\langle X\rangle^{-2-|\alpha|+|\beta|+|\tau|} \leq C_{1}\langle X\rangle^{-1} \in L^{p}\left(\mathbf{R}^{2 d}\right),
$$

when $p>2 d$.

Now let $M>4$, assume that (2.12) holds for $|\alpha+\beta+\tau| \leq M-1$ and we shall prove it for $|\alpha+\beta+\tau|=M$. First we write

$$
X^{\beta+\tau} \partial_{X}^{\alpha} b_{d}=X^{\beta+\tau-\delta} X^{\delta} \partial_{X}^{\alpha-\gamma} \partial_{X}^{\gamma} b_{d}
$$

where we choose $\gamma, \delta$ such that $\gamma \neq 0,|\gamma+\delta|=M-2$ and $|\alpha-\gamma|+$ $|\beta+\tau-\delta|=2$. Then, applying (2.5), we get

$$
\begin{gathered}
\left\|X^{\beta+\tau} \partial_{X}^{\alpha} b_{d}\right\|_{L^{p}\left(\mathbf{R}^{2 d}\right)} \leq\left\|X^{\beta+\tau-\delta}\left[X^{\delta}, \partial_{X}^{\alpha-\gamma}\right] \partial_{X}^{\gamma} b_{d}\right\|_{L^{p}\left(\mathbf{R}^{2 d}\right)} \\
+\left\|X^{\beta+\tau-\delta} \partial_{X}^{\alpha-\gamma}\left(X^{\delta} \partial_{X}^{\gamma} b_{d}\right)\right\|_{L^{p}\left(\mathbf{R}^{2 d}\right)} \\
\leq\left\|X^{\beta+\tau-\delta}\left[X^{\delta}, \partial_{X}^{\alpha-\gamma}\right] \partial_{X}^{\gamma} b_{d}\right\|_{L^{p}\left(\mathbf{R}^{2 d}\right)}+C_{p}\left\|H_{0}\left(X^{\delta} \partial_{X}^{\gamma} b_{d}\right)\right\|_{L^{p}\left(\mathbf{R}^{2 d}\right)} \\
\leq\left\|X^{\beta+\tau-\delta}\left[X^{\delta}, \partial_{X}^{\alpha-\gamma}\right] \partial_{X}^{\gamma} b_{d}\right\|_{L^{p}\left(\mathbf{R}^{2 d}\right)}+C_{p}\left\|\left[H_{0}, X^{\delta} \partial_{X}^{\gamma}\right] b_{d}\right\|_{L^{p}\left(\mathbf{R}^{2 d}\right)},
\end{gathered}
$$

since $X^{\delta} \partial_{X}^{\gamma}\left(H_{0} b_{d}\right)=0$. Here we used the fact that $H_{0} b_{d}=1$ and $\gamma \neq 0$. We now estimate the two terms in the right-hand side of (2.13).

Concerning the first term we have

$$
X^{\beta+\tau-\delta}\left[X^{\delta}, \partial_{X}^{\alpha-\gamma}\right] \partial_{X}^{\gamma} b_{d}=-\sum_{\substack{0 \neq \sigma \leq \alpha-\gamma \\
\sigma \leq \delta}}\left(\begin{array}{c}
\alpha-\gamma \\
\sigma
\end{array}\right) \frac{\delta !}{(\delta-\sigma) !} X^{\beta+\tau-\sigma} \partial_{X}^{\alpha-\sigma} b_{d}
$$

We can now apply the inductive assumption observing that

$$
|\alpha-\sigma|+|\beta+\tau-\sigma|_{10}=M-2|\sigma|<M<1
$$


and that $\delta ! /(\delta-\sigma) ! \leq M^{|\sigma|}$ and we obtain

$$
\begin{gathered}
\left\|X^{\beta+\tau-\delta}\left[X^{\delta}, \partial_{X}^{\alpha-\gamma}\right] \partial_{X}^{\gamma} b_{d}\right\|_{L^{p}\left(\mathbf{R}^{2 d}\right)} \\
\leq \sum_{\substack{0 \neq \sigma \leq \alpha-\gamma \\
\sigma \leq \delta}}\left(\begin{array}{c}
\alpha-\gamma \\
\sigma
\end{array}\right) M^{|\sigma|} C^{M-2|\sigma|+1}(M-2|\sigma|)^{M-2|\sigma|} \\
\leq \sum_{\substack{0 \neq \sigma \leq \alpha-\gamma \\
\sigma \leq \delta}}\left(\begin{array}{c}
\alpha-\gamma \\
\sigma
\end{array}\right) C^{M-2|\sigma|+1} M^{M-|\sigma|} \\
\leq C^{M-1} M^{M-1} \sum_{0 \neq \sigma \leq \alpha-\gamma}\left(\begin{array}{c}
\alpha-\gamma \\
\sigma
\end{array}\right) \\
\leq 2^{|\alpha-\gamma|} C^{M-1} M^{M-1} \leq \frac{4}{C^{2}} C^{M+1} M^{M} \leq \frac{1}{2} C^{M+1} M^{M}
\end{gathered}
$$

if $C$ was chosen larger than $2 \sqrt{2}$.

In order to estimate the second term in the right-hand side of (2.13), we observe that the operator $H_{0}$ is of the form

$$
H_{0}=\sum_{\left|\rho_{1}\right|+\left|\rho_{2}\right| \leq 2} c_{\rho_{1} \rho_{2}} X^{\rho_{2}} \partial_{X}^{\rho_{1}}
$$

for some constants $c_{\rho_{1} \rho_{2}} \in \mathbf{R}$. Moreover we have

$$
\begin{aligned}
& {\left[X^{\rho_{2}} \partial_{X}^{\rho_{1}}, X^{\delta} \partial_{X}^{\gamma}\right] b_{d}} \\
& \quad=\sum_{\substack{0 \neq \sigma \leq \rho_{1} \\
\sigma \leq \delta}} c_{\rho_{1} \delta \sigma}^{1} X^{\delta+\rho_{2}-\sigma} \partial_{X}^{\gamma+\rho_{1}-\sigma} b_{d}-\sum_{\substack{0 \neq \sigma \leq \rho_{2} \\
\sigma \leq \gamma}} c_{\rho_{2} \gamma \sigma}^{2} X^{\delta+\rho_{2}-\sigma} \partial_{X}^{\gamma+\rho_{1}-\sigma} b_{d},
\end{aligned}
$$

where

$$
c_{\rho_{1} \delta \sigma}^{1}=\left(\begin{array}{c}
\rho_{1} \\
\sigma
\end{array}\right) \frac{\delta !}{(\delta-\sigma) !}, \quad c_{\rho_{2} \gamma \sigma}^{2}=\left(\begin{array}{c}
\rho_{2} \\
\sigma
\end{array}\right) \frac{\gamma !}{(\gamma-\sigma) !} .
$$

The constants $c_{\rho_{1} \delta \sigma}^{1}, c_{\rho_{2} \gamma \sigma}^{2}$ are bounded from above by $C_{3} M^{|\sigma|}$ for some constant $C_{3}$. Therefore,

$$
\left\|\left[X^{\rho_{2}} \partial_{X}^{\rho_{1}}, X^{\delta} \partial_{X}^{\gamma}\right] b_{d}\right\|_{L^{p}\left(\mathbf{R}^{2 d}\right)} \leq C_{3}\left(S_{1}+S_{2}\right),
$$

where

$$
S_{1}=\sum_{\substack{0 \neq \sigma \leq \rho_{1} \\ \sigma \leq \delta}} M^{|\sigma|}\left\|X^{\delta+\rho_{2}-\sigma} \partial_{X}^{\gamma+\rho_{1}-\sigma} b_{d}\right\|_{L^{p}\left(\mathbf{R}^{2 d}\right)}
$$

and

$$
S_{2}=\sum_{\substack{0 \neq \sigma \leq \rho_{2} \\ \sigma \leq \gamma}} M^{|\sigma|}\left\|X^{\delta+\rho_{2}-\sigma} \partial_{X}^{\gamma+\rho_{1}-\sigma} b_{d}\right\|_{L^{p}\left(\mathbf{R}^{2 d}\right)} .
$$


In order to estimate $S_{1}$ and $S_{2}$ in (2.15) we observe that $|\gamma|+|\delta|=$ $M-2$ and $\left|\rho_{1}\right|+\left|\rho_{2}\right| \leq 2$ imply

$$
\left|\gamma+\rho_{1}-\sigma\right|+\left|\delta+\rho_{2}-\sigma\right| \leq M-2|\sigma| \text {. }
$$

Then, from the inductive assumption we obtain

$$
\left\|X^{\delta+\tilde{\beta}-\sigma} \partial_{X}^{\gamma+\rho_{1}-\sigma} b_{d}\right\|_{L^{p}\left(\mathbf{R}^{2 d}\right)} \leq C^{M-2|\sigma|+1}(M-2|\sigma|)^{M-2|\sigma|},
$$

giving that

$$
\left\|\left[X^{\rho_{2}} \partial_{X}^{\rho_{1}}, X^{\delta} \partial_{X}^{\gamma}\right] b_{d}\right\|_{L^{p}\left(\mathbf{R}^{2 d}\right)} \leq 2 C_{3} C^{M-1} M^{M} \sum_{1 \leq|\sigma| \leq 2} M^{-|\sigma|} .
$$

By combining (2.13), (2.14) and (2.16), and choosing $C$ sufficiently large, we get

$$
\begin{aligned}
\left\|X^{\beta+\tau} \partial_{X}^{\alpha} b_{d}\right\|_{L^{p}\left(\mathbf{R}^{2 d}\right)} & \leq \frac{1}{2} C^{M+1} M^{M} \\
& +2 C_{p} C_{3} C^{M-1} M^{M} \sum_{\left|\rho_{1}\right|+\left|\rho_{2}\right| \leq 2}\left|c_{\rho_{1} \rho_{2}}\right| \sum_{1 \leq|\sigma| \leq 2} M^{-|\sigma|} \leq C^{M+1} M^{M} .
\end{aligned}
$$

This gives (2.12).

Now by estimate (2.12), standard Sobolev embedding estimates and the fact that $|\alpha|^{|\alpha|} \leq C_{d}^{|\alpha|} \alpha$ !, we get

$$
\left|\partial_{X}^{\alpha} b_{d}(X)\right| \leq C^{|\alpha|+1} \alpha !\langle X\rangle^{-|\alpha|}, \quad X \in \mathbf{R}^{2 d} .
$$

To obtain (0.8) for $|X| \geq 1$, we finally use the fact that by (2.2) we have

$$
b_{d}(X)=\frac{1}{|X|^{2}}-\frac{1}{4} \frac{\Delta_{X} b_{d}(X)}{|X|^{2}}
$$

for $|X| \neq 0$. Here it follows by induction on $|\alpha|$ that

$$
\left|\partial_{X}^{\alpha}\left(\frac{1}{|X|^{2}}\right)\right| \leq C^{|\alpha|+1} \alpha !\langle X\rangle^{-2-|\alpha|}, \quad|X| \geq 1 .
$$

Hence, by differentiating (2.18) and applying (2.17) and (2.19) we obtain (0.8) for $|X| \geq 1$. This concludes the proof.

So far we have only considered the Weyl symbol of $H^{-1}$. In the following we make some remarks on the symbol of $H^{-1}$ with respect to other pseudo-differential calculi. More precisely, let $t \in \mathbf{R}$, and let $b_{d, t}$ be the $t$-symbol of $H^{-1}$, i. e. $b_{d, t}$ is chosen such that $\mathrm{Op}_{t}\left(b_{d, t}\right)=H_{d}^{-1}$ (cf. (1.1)). We have

$$
b_{d, t}(x, \xi)=e^{i \tau\left\langle D_{\xi}, D_{x}\right\rangle} b_{d}(x, \xi), \quad \tau=t-\frac{1}{2}
$$

(cf. [10]), and by straight-forward computations we get

$$
b_{d, t}(x, \xi)=C \tau^{-d} \iint b_{d}(x-y, \xi-\eta) e^{-i\langle y, \eta\rangle / \tau} d y d \eta,
$$


where the right-hand side is considered as an oscillatory integral, and should be interpreted as $b_{d} * \delta_{0}=b_{d}$ when $\tau=0$ (i. e. when $t=1 / 2$, which is the Weyl case). Here the constant $C$ only depends on the dimension.

We have now the following extension of Proposition 2.2, where essentially the condition (0.7) is replaced by

$$
\left|\partial^{\alpha} b_{d, t}(x, \xi)\right| \leq C^{|\alpha|+1}(\alpha !)^{1 / 2}\langle(x, \xi)\rangle^{-2},
$$

Proposition 2.2. Let $b_{d, t}$ be the t-symbol of the inverse to the harmonic oscillator on $\mathbf{R}^{d}$. Then there is a constant $C>0$ such that (0.7)' holds for every $\alpha \in \mathbf{N}^{2 d}$.

Proof. Since the result is the same as Proposition 2.2 when $t=1 / 2$, we may assume that $t \neq 1 / 2$, or equivalently, that $\tau \neq 0$. We have

$$
e^{-i\langle y, \eta\rangle / \tau}=\frac{\left(1-\Delta_{\eta}\right)^{d+1} e^{-i\langle y, \eta\rangle / \tau}}{\left(1+|y|^{2} / \tau^{2}\right)^{d+1}},
$$

and using this in (2.21), and integrating by parts, we get

$$
b_{d, t}(x, \xi)=C \tau^{-d} \iint \frac{\left(\left(1-\Delta_{\xi}\right)^{d+1} b_{d}\right)(x-y, \xi-\eta)}{\left(1+|y|^{2} / \tau^{2}\right)^{d+1}} e^{-i\langle y, \eta\rangle / \tau} d y d \eta .
$$

In the same way we have

$$
e^{-i\langle y, \eta\rangle / \tau}=\frac{\left(1-\Delta_{y}\right)^{d+1} e^{-i\langle y, \eta\rangle / \tau}}{\left(1+|\eta|^{2} / \tau^{2}\right)^{d+1}},
$$

and again integrations by parts give

$$
\begin{array}{r}
b_{d, t}(x, \xi) \\
=C \tau^{-d} \iint\left(1-\Delta_{y}\right)^{d+1}\left(\frac{\left(\left(1-\Delta_{\xi}\right)^{d+1} b_{d}\right)(x-y, \xi-\eta)}{\left(1+|y|^{2} / \tau^{2}\right)^{d+1}}\right) \\
\times \frac{e^{-i\langle y, \eta\rangle / \tau}}{\left(1+|\eta|^{2} / \tau^{2}\right)^{d+1}} d y d \eta \\
=\sum_{|\beta| \leq 4 d} C_{\beta} u_{\beta} * \psi_{\beta},
\end{array}
$$

where $u_{\beta}=\partial^{\beta} b_{d}$, and $\psi_{\beta}(x, \xi)$ are equal to

$$
P_{\beta}(D)\left(\langle x / \tau\rangle^{-2 d-2}\langle\xi / \tau\rangle^{-2 d-2}\right),
$$

for some differential operator $P_{\beta}(D)$ with constant coefficients of order at most $4 d$, and which depend on $\beta$ only. In particular it follows that for some constant $C>0$ we have

$$
\left|\psi_{\beta}(X)\right| \leq C\langle X\rangle^{-2 d-2}
$$

for every $\beta$. 
The result follows if we prove that for every $\beta$, there is a constant $C$ such that we have

$$
\left|\left(\partial^{\alpha} u_{\beta}\right) * \psi_{\beta}\right| \leq C^{1-|\alpha|}(\alpha !)^{1 / 2}\langle X\rangle^{-2} .
$$

We have

$$
\begin{gathered}
\left|\left(\left(\partial^{\alpha} u_{\beta}\right) * \psi_{\beta}\right)(X)\right| \leq\left(\left|\partial^{\alpha} u_{\beta}\right| *\left|\psi_{\beta}\right|\right)(X) \\
\leq C_{1} \sum_{|\beta| \leq 4 d}\left(\left|\partial^{\alpha+\beta} b_{d}\right| *\langle\cdot\rangle^{-2 d-2}\right)(X) \\
\leq C_{2} \sum_{|\beta| \leq 4 d}\left(\mid \partial^{\alpha+\beta} C^{1+|\alpha+\beta|}(\alpha+\beta) !^{1 / 2}\langle\cdot\rangle^{-2} *\langle\cdot\rangle^{-2 d-2}\right)(X) \\
\leq C_{3}^{1+|\alpha|} \alpha !^{1 / 2} \sum_{|\beta| \leq 4 d}\left(\langle\cdot\rangle^{-2} *\langle\cdot\rangle^{-2 d-2}\right)(X) \\
\leq C_{4}^{1+|\alpha|} \alpha !^{1 / 2}\langle X\rangle^{-2},
\end{gathered}
$$

for some constants $C_{k}, k=1, \ldots, 4$, which only depend on $\beta$. This proves the result.

Remark 2.4. The techniques in the preceding proof can also be applied to obtain estimates for $b_{t, d}$, related to Proposition 2.3, where the decay should be similar as in the estimates (0.4)-(0.8). In such approach, one needs to apply the operators

$$
\frac{1-\Delta_{\eta}}{1+|y|^{2} / \tau^{2}} \text { and } \frac{1-\Delta_{y}}{1+|\eta|^{2} / \tau^{2}}
$$

$|\alpha|+2 d+2$ times instead of $2 d+2$. More precisely, if $f_{r}(x)=\langle x\rangle^{-r}$, $x \in \mathbf{R}^{d}$, then it follows by straight-forward computations that

$$
\left|\partial^{\alpha} f_{r_{1}} * f_{r_{2}}\right| \leq C^{r} \alpha ! f_{r+|\alpha|}, \quad r=\min \left(r_{1}, r_{2}\right),
$$

for some constant $C$ which is independent of $r_{1}, r_{2} \in \mathbf{R}$ and $\alpha \in \mathbf{N}^{d}$ such that $\max \left(r_{1}, r_{2}\right) \geq d+1$. Here it seems not possible to replace $r$ by larger values in the inequality (2.27). Consequently, if the functions which corresponds to $\partial^{\alpha} u_{\beta} * \psi_{\beta}$ in the previous proofs should be bounded by functions of the form $\langle X\rangle^{-2-|\alpha|}$, it is required that the operators in (2.26) are applied the asserted number of times.

This has also consequences on the final estimate. In fact, in the expressions which corresponds to (2.25), one obtains one factor $\alpha$ ! from $b_{d}$, because of (10.8), and one such factor because of the factorial in (2.27). Hence, from such computations it follows that $b_{d, t}$ satisfies

$$
\left|\partial^{\alpha} b_{d, t}(x, \xi)\right| \leq C^{|\alpha|+1} \alpha !^{2}\langle(x, \xi)\rangle^{-2-|\alpha|},
$$

for some constant $C$ which is independent of $\alpha$. By taking the geometric mean-value with the previous proposition we obtain

$$
\left|\partial^{\alpha} b_{d, t}(x, \xi)\right| \leq C_{14}^{|\alpha|+1} \alpha !^{(1+3 s) / 2}\langle(x, \xi)\rangle^{-2-s|\alpha|} .
$$


Consequently, the obtained estimates for $b_{d, t}$ when $t \neq 1 / 2$ and $s>0$, are not so strong compared to the Weyl symbol $b_{d}$, when using this method of approximation, since the factor $\alpha !^{(1+3 s) / 2}$ in (2.28) increases faster compared to the factor $\alpha !^{(1+s) / 2}$ in (0.6).

On the other hand, by using more refined methods which also involve symbolic calclulus it is here conjectured that (2.28) can be improved in such way that the factor $\alpha !^{(1+3 s) / 2}$ can be replaced by a factor $\alpha !^{!_{0}}$, for some $s_{0}$ which is strictly smaller than $(1+3 s) / 2$, when $s>0$.

Remark 2.5. Let $t \in \mathbf{R}$, and consider the general harmonic oscillator

$$
H=-\Delta+C_{1}|x|^{2}+C_{2},
$$

which can be found in Remark 1.3. Here $C_{1}, C_{2} \in \mathbf{R}$ should satisfy (1.5). It follows that $H$ is an invertible and globally elliptic operator on $\mathscr{S}$ and $\mathcal{S}_{s}$, and their dual spaces, for every $s \geq 1 / 2$ (cf. e. g. [17, 18]). The inverse $H^{-1}$ of $H$ is a Weyl operator $\mathrm{Op}^{w}\left(b_{d}\right)$ or a $t$-operator $\mathrm{Op}_{\tau}\left(b_{d, t}\right)$, for some appropriate smooth functions $b_{d}$ and $b_{d, t}$ on $\mathbf{R}^{2 d}$.

For such choices of $C_{1}$ and $C_{2}$, it follows by Theorem 2.1 and Proposition 2.2, and their proofs that these results remain valid after the standard harmonic oscillator has been replaced by the operator in (2.29).

\section{EXPLICIT FORMULAS FOR THE INVERSE OF THE HARMONIC OSCILLATOR}

In this section we derive some formulas for the symbol $b_{d}(x, \xi)$ starting from the equation (2.2) As before, let $X=(x, \xi) \in \mathbf{R}^{2 d}$. By a slight dilation of the variables in $b_{d}$, we may reformulate (2.2) as an equation of the form

$$
H_{2 d} F=G,
$$

where $G$ is constant. Hence (0.10) holds for some real valued $c_{d}$, in view of Corollary 1.2. By straight-forward computations, (0.9) and the radial property (0.10), it follows that $c_{d}$ satisfies

$$
-t c_{d}^{\prime \prime}(t)-d c_{d}^{\prime}(t)+t c_{d}(t)=1 .
$$

We see from (3.1) that $c_{d}^{\prime}(0)=-1 / d$. Furthermore, we know that $|X|^{2} b_{d}(X)$ is bounded. This implies that $t c_{d}(t)$ is bounded. Moreover, by Proposition 2.2 it follows that $c_{d}$ is extendable to an entire function. In particular, it is equal to its power series expansion, i. e.

$$
c_{d}(t)=\sum_{k=0}^{\infty} a_{k} t^{k}
$$


for some sequence $\left\{a_{k}\right\}_{k=0}^{\infty}$. By differentiations we have

$$
\begin{aligned}
& t c_{d}^{\prime \prime}(t)=\sum_{k=1}^{\infty}(k+1) k a_{k+1} t^{k}, \quad c_{d}^{\prime}(t)=\sum_{k=0}^{\infty}(k+1) a_{k+1} t^{k} \\
& t c_{d}(t)=\sum_{k=1}^{\infty} a_{k-1} t^{k}
\end{aligned}
$$

By inserting this into (3.2) we get

$$
\sum_{k=1}^{\infty}\left(-(k+1)(k+d) a_{k+1}+a_{k-1}\right) t^{k}-d a_{1}=1,
$$

which gives

$$
a_{1}=-\frac{1}{d}, \quad a_{k}=\frac{a_{k-2}}{k(k+d-1)}, \quad k \geq 2 .
$$

If $k=2 p$ is even, then the latter equation gives

$$
a_{2 p}=\frac{a_{0}}{(2 p) ! !(2 p+d-1)(2 p+d-3) \cdots(d+1)},
$$

and if $k=2 p+1$ is odd, we get

$$
a_{2 p+1}=\frac{a_{1}}{(2 p+1) ! !(2 p+d)(2 p+d-2) \cdots(d+2)} .
$$

This gives

$$
a_{2 p}=\frac{a_{0}(d-1) ! !}{(2 p) ! !(2 p+d-1) ! !}, \quad a_{2 p+1}=\frac{a_{1} d ! !}{(2 p+1) ! !(2 p+d) ! !} .
$$

Here and in what follows we set $0 ! !=1$, as usual.

Since $a_{1}=-1 / d$, we get

$$
\begin{aligned}
& c_{d}(t) \\
& =\frac{d ! !}{d}\left(\alpha \sum_{p=0}^{\infty} \frac{t^{2 p}}{(2 p) ! !(2 p+d-1) ! !}-\sum_{p=0}^{\infty} \frac{t^{2 p+1}}{(2 p+1) ! !(2 p+d) ! !}\right),
\end{aligned}
$$

where $\alpha=a_{0}(d-1) ! ! d / d ! !$. Since $b$ is bounded together with all its derivatives, the same is true for $c_{d}(t)$ when $t \geq 0$. This implies that $\alpha$ is uniquely determined. In fact, the right-hand side of (3.5) is a difference of two sums, which both increase to infinity faster than any polynomial. Hence there is at most one choice of $\alpha$ such that $c_{d}(t)$ is bounded when $t \geq 0$.

We claim that $\alpha$ is independent of $d$ when $d$ stays purely among the even numbers, or purely among the odd numbers. This means that if $\alpha=\alpha_{d}$ in (3.5), then we claim that $\alpha_{d}=\alpha_{d-2}$ for every $d \geq 3$. 
In fact, let $\beta=\alpha_{d}-\alpha_{d-2}$. Then it follows from (3.5) and straightforward computations that

$$
(d-1) c_{d}(t)+t c_{d}^{\prime}(t)-(d-2) c_{d-2}(t)=\beta(d-2) ! ! \sum_{p=0}^{\infty} \frac{t^{2 p}}{(2 p) ! !(2 p+d-3) ! !} .
$$

Here the left-hand side is bounded when $t \geq 0$. Since $(d-2) ! !>0$ and the power series on the right-hand side is unbounded, it follows that $\beta=0$. This proves the stated invariance, as well as

$$
(d-1) c_{d}(t)+t c_{d}^{\prime}(t)=(d-2) c_{d-2}(t), \quad d \geq 3 .
$$

Proposition 3.1. Let $c_{d}$ be such that $c_{d}\left(|X|^{2}\right)$ is the Weyl symbol of the inverse to the harmonic oscillator on $\mathbf{R}^{d}$. Then $c_{d}$ is given by (3.5), where $\alpha=1$ when $d$ is even, and $\alpha=\pi / 2$ when $d$ is odd.

Proof. We may assume that $d=2$ when considering the case when $d$ is even. By (3.5) we have

$$
\begin{aligned}
c_{2}(t)=\alpha \sum_{p=0}^{\infty} \frac{t^{2 p}}{(2 p+1) !}-\sum_{p=0}^{\infty} \frac{t^{2 p+1}}{(2 p+2) !} & \\
= & \frac{1-(\cosh (t)-\alpha \sinh (t))}{t} .
\end{aligned}
$$

Since $c_{2}$ should be bounded at infinity, it follows from the last expression that $\alpha=1$, and the result follows in this case.

Next we consider the case when $d$ is odd, and then we may assume that $d=1$. Let $F$ be the (one-sided) Laplace transform of $c=c_{1}$. Then the Laplace transforms of $t c(t), c^{\prime}(t), t c^{\prime \prime}(t)$ and 1 are

$$
s \mapsto-F^{\prime}(s), \quad s \mapsto s F(s)-c(0), \quad s \mapsto-2 s F(s)-s^{2} F^{\prime}(s)+c(0)
$$

and

$$
s \mapsto \frac{1}{s},
$$

respectively. Hence by Laplace transformation, the equation (3.1) becomes

$$
\left(s^{2}-1\right) F^{\prime}(s)+s F(s)=\frac{1}{s},
$$

and the general solution of this equation is

$$
F(s)=\frac{\arctan \left(\sqrt{s^{2}-1}\right)+C}{\sqrt{s^{2}-1}}, \quad s>1 .
$$

Since $c_{1}(t)$ is bounded for $t \geq 1$, it follows that $F$ is extendable to an analytic function on the half plane $\operatorname{Re}(s)>0$. This implies that $C=0$, and $F(s)$ should be interpreted as

$$
F(s)=\sum_{p=0}^{\infty} \frac{(-1)^{p}\left(s^{2}-1\right)^{p}}{2 p+1}, \quad \text { when } \quad 0 \leq s \leq \sqrt{2}
$$


Summing up, it follows that

$$
F(s)= \begin{cases}\sum_{p=0}^{\infty} \frac{(-1)^{p}\left(s^{2}-1\right)^{p}}{2 p+1}, & 0<s \leq 1, \\ \frac{\arctan \left(\sqrt{s^{2}-1}\right)}{\sqrt{s^{2}-1}}, & s>1 .\end{cases}
$$

Now we get

$$
c_{1}(0)=\lim _{s \rightarrow \infty} s F(s)=\lim _{s \rightarrow \infty} s \cdot \frac{\arctan \left(\sqrt{s^{2}-1}\right)}{\sqrt{s^{2}-1}}=\frac{\pi}{2},
$$

and the result now follows from these equalities and letting $t=0$ in (3.5) . The proof is complete.

It follows from Theorem 2.1 and Faà di Bruno's formula, and the fact $b_{d}(X)=c_{d}\left(|X|^{2}\right)$, that for some constant $C$ we have

$$
\left|c_{d}^{(k)}(t)\right| \leq C^{1+k}(k !)^{(1+s) / 2}(1+t)^{-1-s k}, \quad t \geq 0,
$$

for every $s \in[0,1]$ and $k \in \mathbf{N}$. We shall now go beside the main stream for a while and combine this inequality with (3.5) to establish narrow estimates for the special function

$$
w_{n}(t) \equiv \sum_{p=0}^{\infty} \frac{t^{2 p+1}}{(2 p+1) ! !(2 p+2 n+1) ! !},
$$

in terms of the Bessel function

$$
u_{n}(t) \equiv \sum_{p=0}^{\infty} \frac{t^{p}}{p !(p+n) !} .
$$

In fact, if $d=2 n+1$ is odd, then Proposition 3.1, (3.5) and (3.9) give the following result.

Theorem 3.2. Let $n \in \mathbf{N}, w_{n}(t)$ and $u_{n}(t)$ be as in (3.10) and (3.11). Then

$$
\left|\frac{d^{k}}{d t^{k}}\left(w_{n}(t)-\frac{\pi}{2} u_{n}\left(t^{2} / 4\right)\right)\right| \leq C^{1+k}(k !)^{(1+s) / 2}(1+t)^{-1-s k}, \quad t \geq 0
$$

for some constant $C>0$ which is independent of $s, k$ and $t \geq 0$.

Remark 3.3. Note that the coefficients in the power series in (3.12) contain two factors with odd semi-factorials, which can be formulated by four factors of factorials. It seems to be difficult to find qualitative estimates in the literature for special functions which are obtained by such power series expansion (cf. e. g. [13]). The estimate (3.12) might then shed some light on how such functions can be approximated in terms of the more well-known Bessel functions. 
We now continue with our analysis of $b_{d}$ when $d=2 n$ is even.

From (3.7) we have

$$
c_{2}(t)=\frac{1-e^{-t}}{t}
$$

giving that

$$
b_{2}(X)=\frac{1-e^{-|X|^{2}}}{|X|^{2}}, \quad d=2,
$$

which can also be rewritten as (0.3).

Moreover, by differentiating (3.6) and using (3.1) we obtain the following recursive formula

$$
t c_{d}(t)=(d-2) c_{d-2}^{\prime}(t)+1 .
$$

For example, by (3.13) and (3.15), we get

$$
c_{4}(t)=\frac{2(t+1) e^{-t}+t^{2}-2}{t^{3}} .
$$

Hence,

$$
b_{4}(X)=\frac{2\left(|X|^{2}+1\right) e^{-|X|^{2}}+|X|^{4}-2}{|X|^{6}}, \quad d=4 .
$$

Now we aim to prove a general compact formula for $b_{d}(X)$ in the even dimensional case. To this hand, rather than applying the recursive formula (3.15), we shall proceed by giving first the asymptotic expansion of $c_{d}(t)$ and $b_{d}(X)$, for any $d \geq 1$, in terms of homogeneous functions. In principle, these computations are included in [16, Section 25], but here we need a more explicit result.

Proposition 3.4. Let $c_{d}$ be defined by (0.10), and let $h_{d, j}(t)$ be given by

$$
h_{d, 0}(t)=t^{-1}
$$

and

$$
h_{d, j}(t)=\left((-1)^{j}(2 j-1) ! ! \prod_{l=1}^{j}(d-2 l)\right) t^{-1-2 j}, \quad j \geq 1 .
$$

Then for every $N \in \mathbf{N}, N \geq 1$ and $n \in \mathbf{N}$, there exists a positive constant $C_{n, N}$ such that

$$
\left|\frac{d^{n}}{d t^{n}}\left(c_{d}(t)-\sum_{j<N} h_{d, j}(t)\right)\right| \leq C_{n, N} t^{-1-2 N-n} .
$$

To prove the proposition we need some preliminary results. First we note that by (0.4) and (0.10), it follows that for every $n \geq 0$, there is a constant $C_{n}$ such that

$$
\left|c_{d}^{(n)}(t)\right| \leq C_{n} t^{-1-n}
$$


Lemma 3.5. Let $h_{d, j}$ be the same as in Proposition 3.4. Then

$$
t h_{d, j}(t)=t h_{d, j-1}^{\prime \prime}(t)+d h_{d, j-1}^{\prime}(t), \quad j \geq 1 .
$$

Lemma 3.5 follows by straight-forward computation. The details are left to the reader.

Lemma 3.6. Let $c_{d}$ and $h_{d, j}$ be the same as in Proposition 3.4. If $N \geq 1$, then

$$
\begin{aligned}
t\left(c_{d}(t)\right. & \left.-\sum_{j \leq N} h_{d, j}(t)\right) \\
= & t\left(c_{d}^{\prime \prime}(t)-\sum_{j \leq N-1} h_{d, j}^{\prime \prime}(t)\right)+d\left(c_{d}^{\prime}(t)-\sum_{j \leq N-1} h_{d, j}^{\prime}(t)\right) .
\end{aligned}
$$

Proof. We prove the lemma by induction on $N$. As $t h_{0}(t)=1$, from (3.1) we get

$$
t\left(c_{d}(t)-h_{d, 0}(t)\right)=t c_{d}^{\prime \prime}(t)+d c_{d}^{\prime}(t) .
$$

By Lemma 3.5 we obtain

$$
\begin{aligned}
t\left(c_{d}(t)-h_{d, 0}(t)-h_{d, 1}(t)\right) & =t c_{d}^{\prime \prime}(t)+d c_{d}^{\prime}(t)-t h_{d, 1}(t) \\
=t c_{d}^{\prime \prime}(t)+d c_{d}^{\prime}(t)-t h_{d, 0}^{\prime \prime}(t)-d h_{d, 0}^{\prime}(t) & \\
& =t\left(c_{d}^{\prime \prime}(t)-h_{d, 0}^{\prime \prime}(t)\right)+d\left(c_{d}^{\prime}(t)-h_{d, 0}^{\prime}(t)\right),
\end{aligned}
$$

which gives the assertion for $N=1$.

Assume now that (3.21) is true for some $N$ and let us prove it for $N+1$. By (3.20) and by the inductive assumption we get

$$
\begin{gathered}
t\left(c_{d}(t)-\sum_{j \leq N+1} h_{d, j}(t)\right)=t\left(c_{d}(t)-\sum_{j \leq N} h_{d, j}(t)\right)-t h_{d, N+1}(t) \\
=t\left(c_{d}^{\prime \prime}(t)-\sum_{j \leq N-1} h_{d, j}^{\prime \prime}(t)\right)+d\left(c_{d}^{\prime}(t)-\sum_{j \leq N-1} h_{d, j}^{\prime}(t)\right)-t h_{d, N}^{\prime \prime}(t)-d h_{d, N}^{\prime}(t) \\
=t\left(c_{d}^{\prime \prime}(t)-\sum_{j \leq N} h_{d, j}^{\prime \prime}(t)\right)+d\left(c_{d}^{\prime}(t)-\sum_{j \leq N} h_{d, j}^{\prime}(t)\right) .
\end{gathered}
$$

This proves the lemma.

Proof of Proposition 3.4. First let $N=1$. By (3.22) we have

$$
\begin{aligned}
\frac{d^{n}}{d t^{n}}\left(c_{d}(t)-h_{d, 0}(t)\right) & =\frac{d^{n}}{d t^{n}}\left(c_{d}^{\prime \prime}(t)+d \frac{c_{d}^{\prime}(t)}{t}\right) \\
& =c_{d}^{(n+2)}(t)+d \sum_{m \leq n}\left(\begin{array}{c}
n \\
m
\end{array}\right)(-1)^{m} \frac{m !}{t^{m+1}} c_{d}^{(n-m+1)}(t) .
\end{aligned}
$$


Hence (3.19) gives

$$
\left|\frac{d^{n}}{d t^{n}}\left(c_{d}(t)-h_{d, 0}(t)\right)\right| \leq C_{1} t^{-3-n}+2^{n} d C_{2} n ! t^{-3-n} \leq C_{3} t^{-3-n},
$$

for some constants $C_{1}, C_{2}, C_{3}$, and (3.18) follows for $N=1$. For $N>1$ we argue by induction using Lemma 3.6. By (3.21) we have

$$
\begin{aligned}
\frac{d^{n}}{d t^{n}}\left(c_{d}(t)\right. & \left.-\sum_{j \leq N} h_{d, j}(t)\right)=\frac{d^{n}}{d t^{n}}\left(c_{d}^{\prime \prime}(t)-\sum_{j \leq N-1} h_{d, j}^{\prime \prime}(t)\right) \\
& +d \sum_{m \leq n}\left(\begin{array}{c}
n \\
m
\end{array}\right)(-1)^{m} \frac{m !}{t^{m+1}} \frac{d^{n-m}}{d t^{n-m}}\left(c_{d}^{\prime}(t)-\sum_{j \leq N-1} h_{d, j}^{\prime}(t)\right) .
\end{aligned}
$$

By the inductive assumption we get

$$
\left|\frac{d^{n}}{d t^{n}}\left(c_{d}(t)-\sum_{j \leq N} h_{d, j}(t)\right)\right| \leq C t^{-3-2 N-n}
$$

for some constant $C$. This gives the result.

Note that if $d$ is even, we have $h_{d, j}=0$ for $j \geq d / 2$. One cannot expect however that $c_{d}(t)=\sum_{j=0}^{\infty} h_{d, j}(t)$, since the terms in the sum have singularities at the origin. Inspired by (3.13), (3.16), we now define

$$
\widetilde{h}_{d, j}(t)=\left(1-e^{-t} p_{2 j}(t)\right) h_{d, j}(t), \quad j \geq 0,
$$

where $p_{j}(t)$ is the Taylor polynomial of $e^{t}$ of order $j$ centered at $t=0$ and the functions $h_{d, j}(t)$ are the same as in Proposition 3.4. Since terms with exponential decay do not change the asymptotic expansion, we have for some positive constants $C_{n, N}$ the following

$$
\left|\frac{d^{n}}{d t^{n}}\left(c_{d}(t)-\sum_{j<N} \widetilde{h}_{d, j}(t)\right)\right| \leq C_{n, N}\langle t\rangle^{-1-2 N-n}
$$

The singularities at the origin are now cancelled. In the even dimensional case we still have $\widetilde{h}_{d, j}(t)=0$ for $j \geq d / 2$, and the asymptotic expansion (3.24) becomes indeed an identity as proved below.

Proposition 3.7. Let $d=2 n>0$ be even, and let $\widetilde{h}_{d, j}$ be defined by (3.23). Then

$$
c_{d}(t)=\sum_{j=0}^{\infty} \widetilde{h}_{d, j}(t)=\sum_{j=0}^{n-1}\left(\begin{array}{c}
n-1 \\
j
\end{array}\right)(-1)^{j}(2 j) ! \frac{1-e^{-t} p_{2 j}(t)}{t^{2 j+1}} .
$$

Proof. We shall prove the result by induction. First we perform some investigations about the sums in (3.25). We note that $\widetilde{h}_{d, j}=0$ when $j \geq n$, and by straight-forward computations it follows that the second equality in (3.25) hold. 
Let $\varphi_{d}(t)$ be the right-hand side of (3.25). By straight-forward computations we get

$$
\begin{aligned}
\varphi_{d}(t) & =\sum_{j=0}^{n-1}\left(\begin{array}{c}
n-1 \\
j
\end{array}\right)(-1)^{j}(2 j) ! g_{j}(t), \quad \text { where } \quad g_{j}(t)=\frac{1-e^{-t} p_{2 j}(t)}{t^{2 j+1}} \\
\widetilde{h}_{d, j}(t) & =\left(\begin{array}{c}
n-1 \\
j
\end{array}\right)(-1)^{j}(2 j) ! g_{j}(t), \quad \text { and } \quad t g_{j}^{\prime}(t)=-(2 j+1) g_{j}(t)+\frac{e^{-t}}{(2 j) !} .
\end{aligned}
$$

This gives

$$
\begin{aligned}
& t \varphi_{d}^{\prime}(t)+(d-1) \varphi_{d}(t) \\
& =\sum_{j=0}^{n-1}(2 n-2 j-2)\left(\begin{array}{c}
n-1 \\
j
\end{array}\right)(-1)^{j}(2 j) ! g_{j}(t)+e^{-t} \sum_{j=0}^{n-1}\left(\begin{array}{c}
n-1 \\
j
\end{array}\right)(-1)^{j} \\
& \quad=2(n-1) \sum_{j=0}^{n-2}\left(\begin{array}{c}
n-2 \\
j
\end{array}\right)(-1)^{j}(2 j) ! g_{j}(t)+e^{-t} \sum_{j=0}^{n-1}\left(\begin{array}{c}
n-1 \\
j
\end{array}\right)(-1)^{j} .
\end{aligned}
$$

Here the first sum on the right-hand side is $(d-2) \varphi_{d-2}(t)$, and the second sum is zero, by the binomial theorem.

Hence

$$
t \varphi_{d}^{\prime}(t)+(d-1) \varphi_{d}(t)=(d-2) \varphi_{d-2}(t),
$$

that is, the sequence $\left\{\varphi_{2 n}\right\}_{n \geq 1}$ satisfies the same type of differential equations as $\left\{c_{2 n}\right\}_{n \geq 1}$ (cf. (3.6) ). In particular, if $\psi_{d}=c_{d}-\varphi_{d}$, then $\left\{\psi_{2 n}\right\}_{n \geq 1}$ also fulfills (3.6), after $c_{d}$ and $c_{d-2}$ have been replaced by $\psi_{d}$ and $\psi_{d-2}$, respectively.

We now turn into the induction step (over $n$ ). By the definitions, the result follows if we prove that $\psi_{d}=0$ for every $d=2 n$. The result is true for $n=1$, in view of (3.13) and by the definition of $\varphi_{2}$.

Assume that the result is true for $n-1$, i. e. $\psi_{2 n-2}=0$. Then (3.6) implies that $t \psi_{2 n}^{\prime}+(2 n-1) \psi_{2 n}=0$, giving that

$$
\psi_{2 n}(t)=C t^{1-2 n}, \quad t>0,
$$

for some constant $C$. Since $\psi_{2 n}(t)$ is continuous for all $t$ and $t^{1-2 n}$ is singular at origin, it follows that $C$ must be zero, i. e. $\psi_{2 n}=0$. The proof is complete.

Returning now to $b_{d}(X)$, we may reformulate Proposition 3.4 as follows.

Theorem 3.8. Let $b_{d, j}, j=0,1, \ldots$, be given by

$$
b_{d, 0}(X)=|X|^{-2}
$$

and

$$
b_{d, j}(X)=\left((-1)^{j}(2 j-1) ! ! \prod_{\substack{l=1 \\ 22}}^{j}(d-2 l)\right)|X|^{-2-4 j}, \quad j \geq 1 .
$$


Then, for every $N \in \mathbf{N}, N \geq 1$ and for every $\alpha \in \mathbf{N}^{2 d}$ the following estimate holds:

$$
\left|\partial_{X}^{\alpha}\left(b_{d}(X)-\sum_{j<N} b_{d, j}(X)\right)\right| \leq C_{\alpha, N}|X|^{-2-4 N-|\alpha|}
$$

for some positive constant $C_{\alpha, N}$ depending only on $\alpha, N$ and on the dimension $d$.

Finally, by Proposition 3.7 we get the following result which gives exact formulas for $b_{d}$ when $d$ is even.

Theorem 3.9. Let $d=2 n>0$ be even, and let $p_{j}$ be the Taylor polynomial of $e^{t}$ of order $j$ centered at $t=0$. Then

$$
b_{2 n}(X)=\sum_{j=0}^{n-1}\left(\begin{array}{c}
n-1 \\
j
\end{array}\right)(-1)^{j}(2 j) ! \frac{1-e^{-|X|^{2}} p_{2 j}\left(|X|^{2}\right)}{|X|^{2+4 j}} .
$$

\section{REFERENCES}

[1] J.M. Bony, J.Y. Chemin, Espaces fonctionnels associés au calcul de WeylHörmander, Bull. Sci. Math. France 122 (1994), 77-118.

[2] L. Boutet de Monvel, P. Krée, Pseudodifferential operators and Gevrey classes, Ann. Inst Fourier, Grenoble 17 (1967), 295-323.

[3] M. Cappiello, T. Gramchev, L. Rodino, Super-exponential decay and holomorphic extensions for semilinear equations with polynomial coefficients, J. Funct. Anal. 237 (2006), 634-654.

[4] M. Cappiello, T. Gramchev, L. Rodino, Entire extensions and exponential decay for semilinear elliptic equations. J. Anal. Math. 111 (2010), 339-367.

[5] M. Cappiello, T. Gramchev and L. Rodino, Sub-exponential decay and uniform holomorphic extensions for semilinear pseudodifferential equations. Comm. Partial Differential Equations 35 (2010) n. 5, 846-877.

[6] M. Cappiello, L. Rodino, SG-pseudodifferential operators and Gelfand-Shilov spaces, Rocky Mountain J. Math. 36 (2006) 4, 1117-1148.

[7] A. Erdélyi, W. Magnus, F. Oberhettinger, F.G. Tricomi, Higher transcendental functions, 2, Bateman Project, California Inst. Technology, McGraw-Hill, 1953.

[8] I. M. Gelfand, G. E. Shilov, Generalized functions, II-III, Academic Press, NewYork London, 1968.

[9] B. Helffer, Théorie spectrale pour des opérateurs globalement elliptiques, Astérisque 112, Soc. Math. de France, 1984.

[10] L. Hörmander The Analysis of Linear Partial Differential Operators, vol I-III, Springer-Verlag, Berlin Heidelberg NewYork Tokyo, 1983, 1985.

[11] M. Langerbruch, Hermite functions and weighted spaces of generalized functions, Manuscripta Math. 119 (2006), 269-285.

[12] F. Nicola, L. Rodino Global pseudo-differential calculus on Euclidean spaces, Pseudo-Differential Operators. Theory and Applications 4 Birkhäuser Verlag, Basel, 2010.

[13] F. W. J. Olver, Asymptotics and special functions Reprint of the 1974 original, Academic Press, New York, 1997.

[14] S. Pilipović, Generalization of Zemanian spaces of generalized functions which elements have series expansion, SIAM J. Math. Anal. 17 (1986), 477-484.

[15] M. Reed, B. Simon, Methods of modern mathematical physics, 1, Academic Press Inc., San Diego, 1980. 
[16] M. Shubin, Pseudodifferential operators and the spectral theory, Springer Series in Soviet Mathematics, Springer Verlag, Berlin 1987.

[17] M. Signahl, J. Toft Mapping properties for the Bargmann transform on modulation spaces, J. Pseudo-Differ. Oper. Appl. 3 (2012), 1-30.

[18] J. Toft, The Bargmann transform on modulation and Gelfand-Shilov spaces, with applications to Toeplitz and pseudo-differential operators, J. PseudoDiffer. Oper. Appl. 3 (2012), 145-227.

[19] M.W. Wong, Weyl transform, Springer-Verlag, Berling, 1988.

Dipartimento di Matematica "G. Peano", Università di Torino, Via Carlo Alberto 10, Torino, Italy

E-mail address: marco.cappiello@unito.it

Dipartimento di Matematica "G. Peano", Università di Torino, Via Carlo Alberto 10, Torino, Italy

E-mail address: luigi.rodino@unito.it

Department of Mathematics, Linnæus University, Växjö, Sweden

E-mail address: joachim.toft@lnu.se 